\title{
Ionic Modulation of Calcium Dynamics in Simulated Human Heart Failure
}

\author{
Maria Teresa Mora, Jose M Ferrero, Beatriz Trenor \\ Universitat Politècnica de València, Valencia, Spain
}

\begin{abstract}
Calcium mishandling observed in failing hearts is responsible for contractile dysfunction and arrhythmias. Thereby, calcium dynamics becomes an important target to explore for the restoration of the altered excitationcontraction coupling. In this study, we performed an insilico sensitivity analysis to understand the mechanisms modulating calcium dynamics in failing human hearts. Using a modified version of the O'Hara et al. human ventricular action potential model, we simulated different failing conditions for every ionic parameter remodeled in heart failure. Calcium biomarkers from simulated endocardial cells, such as systolic and diastolic calcium concentration, were measured and analyzed. The results of this study highlight the importance of maintaining SERCA activity in its normal levels to improve contractility and point to other crucial parameters that also alter calcium handling, such as NCX.
\end{abstract}

\section{Introduction}

Heart failure (HF), the final consequence of diverse cardiovascular pathologies, is an increasing worldwide health problem characterized by contractility problems and malignant arrhythmias. Abnormalities in calcium handling have been associated with this mechanical dysfunction [1], since intracellular calcium is the main regulator of myocardial excitation-contraction coupling.

When studying the electrophysiology of failing hearts, several investigations have explored the mechanisms that give rise to arrhythmogenesis. These studies highlight action potential (AP) prolongation, as well as intracellular $\mathrm{Na}^{+}$accumulation and altered $\mathrm{Ca}^{2+}$ handling [2-4], as the main causes. Further investigation has revealed that, in $\mathrm{HF}$, ionic channels undergo electrical remodelling, i.e. enhancement or loss of function of some transmembrane currents, leading to the electrophysiological alterations observed in failing myocytes.

The extensive literature focusing on the effects of ionic remodeling on AP hypothesizes on potential therapies to avoid or reduce the progress of HF. In this way, restoring calcium handling should be a main target, which is not usually considered. Experimental observations in failing myocytes have quantified reduced calcium transients (CaT) with slow relaxation rates, and a general decrease in $\left[\mathrm{Ca}^{2+}\right]$ inside the cell, affecting the sarcoplasmic reticulum (SR) $\mathrm{Ca}^{2+}$ load [4-6]. To target these pathways, a better knowledge of the ionic mechanisms leading to the aforementioned alterations is required.

The complexity of the cellular electrophysiological system has required the use of a more systematic methodology, able to complement and explain experimental observations. Computer simulations arise as a very helpful tool in the study of cellular electrical mechanisms [7], and this has led to the development of mathematical models of human AP.

In the present study, a sensitivity analysis was performed in a human model of failing myocytes to elucidate the ionic mechanisms that alter intracellular calcium regulation. The effect of different ionic parameters on calcium biomarkers has revealed the influence in modulating calcium handling and possible targets for restoring concentrations and improving contractility.

\section{Methods}

We carried out simulations of human ventricular endocardial cells paced at $1 \mathrm{~Hz}$. The model used was a modified version of the human action potential model by O'Hara et al. (ORd), which includes a detailed formulation of calcium dynamics [8]. We introduced different modifications to improve the sodium current formulation. These changes include an enhancement of $\mathrm{I}_{\mathrm{Na} \text {,fast }}$, changing the dynamics of the gates $m_{s s}, h_{s s}$, and $j_{s s}$ [9], and in an increase of $\mathrm{I}_{\mathrm{NaL}}$ to match experimental observations [10]. On this new model (ORdmm), we introduced ionic remodeling to simulate $\mathrm{HF}$ according to Gomez et al. [11].

The sensitivity analysis of the HF model was performed applying a one-at-a-time variation in the remodeled ionic parameters. On the basic HF model, these parameters were varied to their normal values ("no change"), as in the ORdmm model, and to $\pm 50 \%$ of their HF remodeling condition. All simulations were run until achieving steadystate, before measuring the electrophysiological characteristics. The measured biomarkers included systolic and diastolic $\left[\mathrm{Ca}^{2+}\right]_{\mathrm{i}}, \mathrm{Ca}^{2+}$ transient $(\mathrm{CaT})$ duration at $30 \%$ and $80 \%$ recovery $\left(\mathrm{CaTD}_{30}\right.$ and $\left.\mathrm{CaTD}_{80}\right)$, and $10 \%$ to $90 \%$ CaT rise time $\left(\mathrm{t}_{10-90}\right)$. Other important concentrations of 


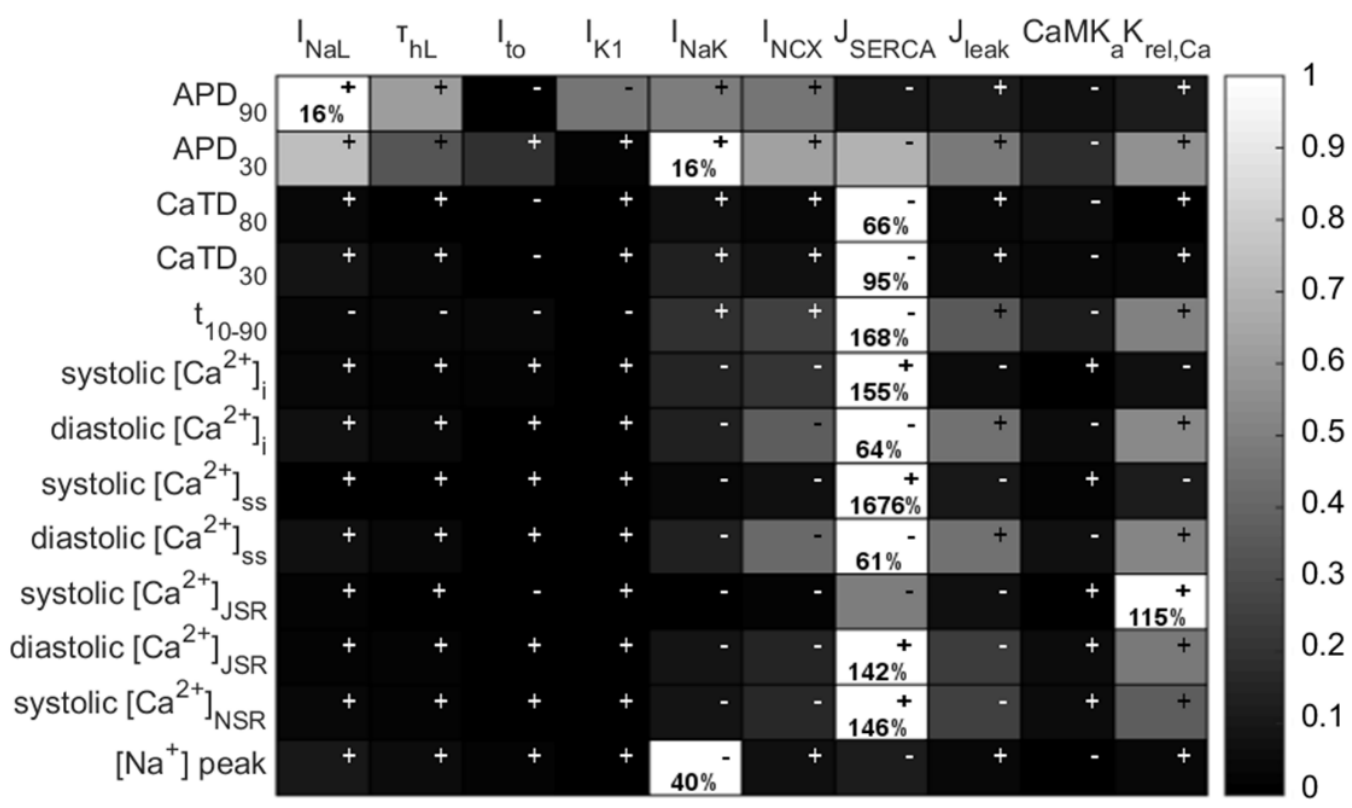

Figure 1. Relative sensitivities of the electrophysiological properties to changes in ionic current parameters in the HF model.

calcium are those reached in the subspace, and in the sarcoplasmic reticulum (SR), divided into the junctional (JSR) and network SR (NSR). In addition, AP duration (APD) was measured at $30 \%$ and $90 \%$ repolarization $\left(\mathrm{APD}_{30}\right.$ and $\left.\mathrm{APD}{ }_{90}\right)$, as well as the $\left[\mathrm{Na}^{+}\right]_{\mathrm{i}}$ peak.

The sensitivity between the most severe HF condition and the "no change" condition was calculated, as described in Trenor et al. [12]. Finally, sensitivities were normalized to the maximum absolute sensitivity for each biomarker.

\section{Results and Discussion}

Figure 1 summarizes the relative sensitivities for all combinations of electrophysiological (EP) characteristics and parameters modified. White indicates the strongest sensitivity per row, while black means lack of dependency between both variables. Signs denote a direct $(+)$ or inverse (-) relationship. At first glance, white color in $\mathrm{J}_{\text {SERCA }}$ column, in comparison with the rest of parameters, highlights its high contribution to most of the EP properties related to calcium handling.

APD and $\left[\mathrm{Na}^{+}\right]_{i}$ have also been analyzed because of the important changes undergone in HF. Figure 2 shows the modulation of $\mathrm{APD}_{90}$ by $\mathrm{I}_{\mathrm{NaL}}, \tau_{\mathrm{hL}}, \mathrm{I}_{\mathrm{NaK}}$ and $\mathrm{I}_{\mathrm{NCX}}$. Failing conditions of these parameters result in a prolongation of APD, with the exception of $\mathrm{I}_{\mathrm{NaK}}$. Our results agree with the abnormal repolarization in myocytes with enhanced $\mathrm{I}_{\mathrm{NaL}}$ [13]. The reduction of $\mathrm{I}_{\mathrm{NaK}}$ is also the main responsible for $\left[\mathrm{Na}^{+}\right]_{\mathrm{i}}$ accumulation (see table 1, last row). High $\mathrm{Na}^{+}$ content activates the reverse mode of the NCX, resulting in APD shortening [14]. However, in failing conditions, enhanced $\mathrm{I}_{\mathrm{NCX}}$ produces APD prolongation due to the exchanger functioning in the forward mode.

Figure 3 shows how the main calcium biomarkers (systolic and diastolic $\left[\mathrm{Ca}^{2+}\right]_{\mathrm{i}}$ ) are modulated by different ionic parameters under different failing conditions. Analyzing the changes in every EP property related to calcium (Figure 1), SR Ca ${ }^{2+}$ ATPase (SERCA) remodeling in HF has negative effects in all of them. Hence, reduced uptake decreases systolic and increases diastolic $\left[\mathrm{Ca}^{2+}\right]$, both in the cytosol (see also Figure 3) and the subspace, lengthens the rise time and duration of $\mathrm{CaT}$, and reduces the SR $\mathrm{Ca}^{2+}$ content. These characteristics have been experimentally observed in human failing myocytes $[4,6]$, but also in healthy hearts when SERCA was inhibited $[15,16]$.

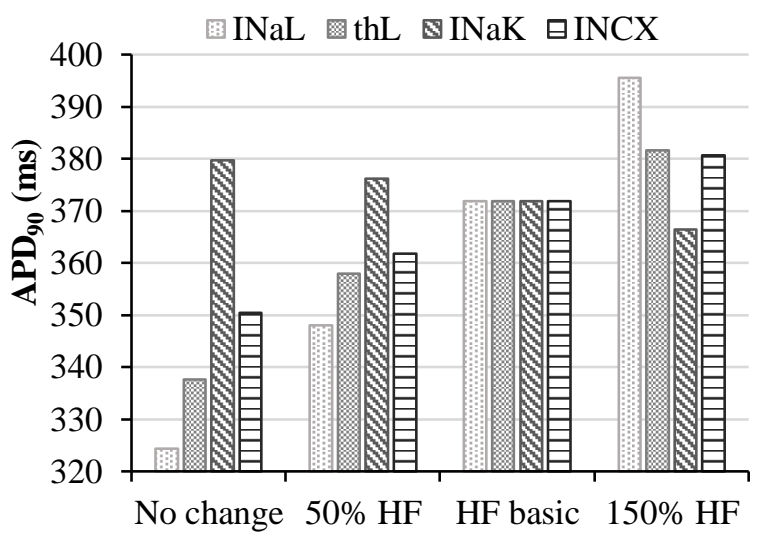

Figure 2. Changes in $\mathrm{APD}_{90}$ with the most influential ionic parameters at different failing conditions. 

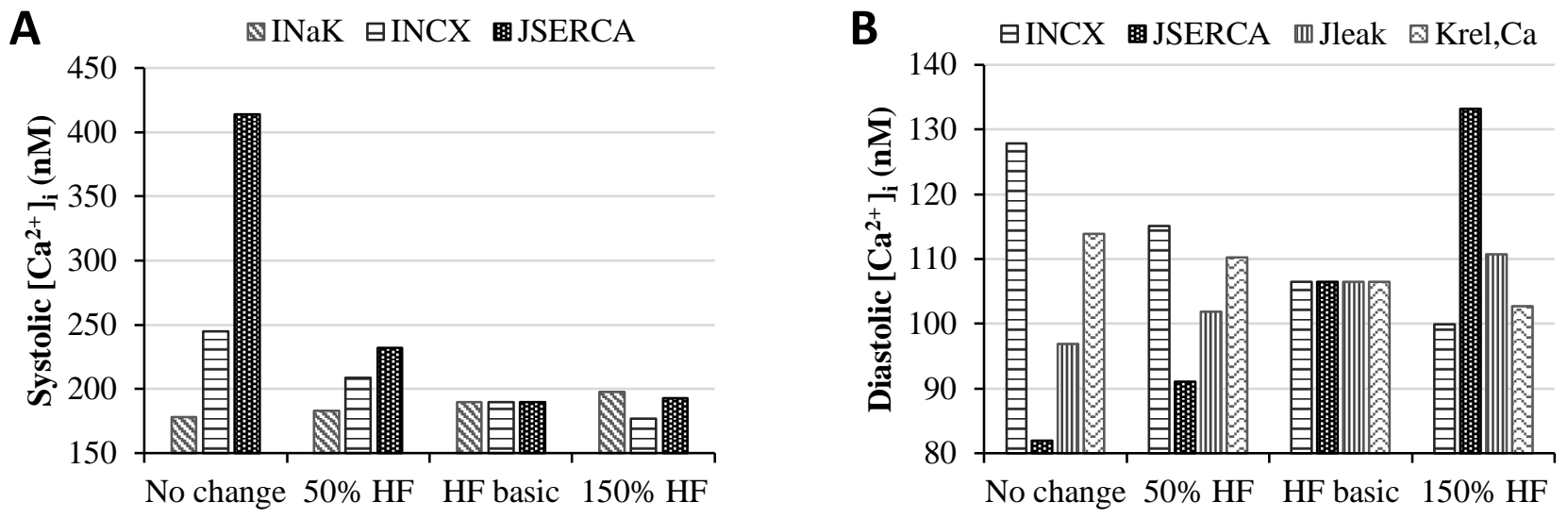

Figure 3. Changes in systolic (panel A) and diastolic $\left[\mathrm{Ca}^{2+}\right]_{i}$ (panel B) with the most influential ionic parameters at different failing conditions.

The next parameters that show some influence in different EP characteristics are those related with $\mathrm{Ca}^{2+}$ release $\left(\mathrm{K}_{\text {rel,Ca }}\right.$ and $\left.\mathrm{J}_{\text {leak }}\right)$. In failing conditions, the former decreases $t_{10-90}$ and diastolic calcium, while the latter increases them, having negative effects. Moreover, both contribute to the reduction of the $\mathrm{SR} \mathrm{Ca}{ }^{2+}$ load. It has been observed that induced Ca-leak modifies CaT negatively [15]. On the other hand, an increase in SR $\mathrm{Ca}^{2+}$ content inhibits RyR leak [17].

According to Figure 1, the following significant sensitivities highlight the strong effect of $\mathrm{I}_{\mathrm{NCX}}$. Although these sensitivities present lower values than some of the sensitivities to the aforementioned parameters, a pronounced remodeling of the NCX exchanger in HF potentiates the changes in some EP characteristics (Figure $3)$. Its negative effects in increasing $t_{10-90}$ and decreasing systolic $\mathrm{Ca}^{2+}$ peak should be highlighted. On the other hand, it decreases diastolic $\left[\mathrm{Ca}^{2+}\right]_{\mathrm{i}}$. Overexpression of NCX in transgenic mice produced a higher $\mathrm{CaT}$ amplitude as well as SR $\mathrm{Ca}^{2+}$ content [18]

Upon these results, abnormalities in $\mathrm{Ca}^{2+}$ handling are mainly related to SERCA dysfunction. Its significantly reduced activity in HF compromises the mechanism of $\mathrm{Ca}^{2+}$ uptake into the SR. In addition, $\mathrm{Ca}^{2+}$ extrusion from the cell by NCX is enhanced, hindering the uptake into the SR. Both mechanisms give rise to a negative balance in the calcium influx-efflux process. Although remodeled RyR channels seem to improve $\mathrm{CaT}$, reduced $\mathrm{SR} \mathrm{Ca}^{2+}$ content decreases the amount of calcium available for release. In the case of $\mathrm{J}_{\text {leak }}, \mathrm{Ca}^{2+}$ release has negative effects because $\mathrm{Ca}^{2+}$ cycling is altered by this uncontrolled factor. To reduce this leak, it would be necessary to know its exact origin. When it is linked to RyR channels, it is difficult to inhibit the leak without affecting the normal process of $\mathrm{Ca}^{2+}$ release. Therefore, further work is required to understand these mechanisms and their consequences.

In view of the global results, the model suggests that, in order to restore calcium dynamics, the first ionic transport to be targeted in HF should be SERCA. In this way, overexpression in failing myocytes cells had good results on $\mathrm{Ca}^{2+}$ handling in rats [19] but also in humans [20], because modified failing cells showed no differences with the healthy ones after the treatment. What is more, it seems not to affect APD.

NCX is another possible target but, unlike SERCA, the inhibition should be done carefully. Inhibiting the exchanger would be beneficial to APD and systolic function, but raising diastolic $\left[\mathrm{Ca}^{2+}\right]_{\mathrm{i}}$ the relaxation might be compromised.

Finally, the one-at-a-time methodology can miss the detection of multifactorial effects and is a limitation of our study. In this sense, a multivariable study to investigate the interaction between different variables and its possible implications would be desirable.

\section{Conclusion}

The major finding of this study is the high modulation of calcium handling by remodeled SERCA. Its influence suggests that repairing $\mathrm{Ca}^{2+}$ uptake function could restore normal calcium dynamics in failing myocytes.

\section{Acknowledgements}

This work was partially supported by Dirección General de Política Científica de la Generalitat Valenciana (PROMETEO 2016/088), and by the "Plan Estatal de Investigación Científica y Técnica y de Innovación 20132016" from the Ministerio de Economía, Industria y Competitividad of Spain (DPI2016-75799-R) and AEI/FEDER, UE.

\section{References}

[1] Sjaastad I, Wasserstrom JA, Sejersted OM. Heart failure a challenge to our current concepts of excitation- 
contraction coupling. J Physiol. 2003;546(Pt 1):33-47.

[2] Drouin E, Lande G, Charpentier F. Amiodarone reduces transmural heterogeneity of repolarization in the human heart. J Am Coll Cardiol. 1998;32(4):1063-7.

[3] Li GR, Lau CP, Leung TK, Nattel S. Ionic current abnormalities associated with prolonged action potentials in cardiomyocytes from diseased human right ventricles. Heart Rhythm. 2004;1(4):460-8.

[4] Piacentino V, Weber CR, Chen X, Weisser-Thomas J, Margulies KB, Bers DM, et al. Cellular basis of abnormal calcium transients of failing human ventricular myocytes. Circ Res. 2003;92(6):651-8.

[5] Bers DM. Altered cardiac myocyte Ca regulation in heart failure. Physiology. 2006;21(17):380-7.

[6] Lou Q, Fedorov VV., Glukhov AV., Moazami N, Fast VG, Efimov IR. Transmural heterogeneity and remodeling of ventricular excitation- contraction coupling in human heart failure. Circulation. 2011;123(17):1881-90.

[7] Gomez JF, Cardona K, Trenor B. Lessons learned from multi-scale modeling of the failing heart. J Mol Cell Cardiol. 2015;89:146-59.

[8] O'Hara T, Virág L, Varró A, Rudy Y. Simulation of the undiseased human cardiac ventricular action potential: Model formulation and experimental validation. PLoS Comput Biol. 2011;7(5).

[9] Passini E, Mincholé A, Coppini R, Cerbai E, Rodriguez B, Severi $\mathrm{S}$, et al. Mechanisms of pro-arrhythmic abnormalities in ventricular repolarisation and antiarrhythmic therapies in human hypertrophic cardiomyopathy. J Mol Cell Cardiol. 2016;96:72-81.

[10] Maltsev VA, Undrovinas AI. A multi-modal composition of the late $\mathrm{Na}+$ current in human ventricular cardiomyocytes. Cardiovasc Res. 2006;69(1):116-27.

[11] Gomez JF, Cardona K, Romero L, Ferrero JM, Trenor B. Electrophysiological and structural remodeling in heart failure modulate arrhythmogenesis. 1D simulation study. PLoS One. 2014;9(9).

[12] Trenor B, Cardona K, Gomez JF, Rajamani S, Ferrero JM, Belardinelli L, et al. Simulation and mechanistic investigation of the arrhythmogenic role of the late sodium current in human heart failure. PLoS One. 2012;7(3):11-3.

[13] Undrovinas AI, Belardinelli L, Undrovinas NA, Sabbah HN. Ranolazine improves abnormal repolarization and contraction in left ventricular myocytes of dogs with heart failure by inhibiting late sodium current. J Cardiovasc Electrophysiol. 2006;17(Suppl 1):S169-77.

[14] Levi AJ, Dalton GR, Hancox JC, Mitcheson JS, Issberner J, Bates JA, et al. Role of intracellular sodium overload in the genesis of cardiac arrhythmias. J Cardiovasc Electrophysiol. 1997;8(6):700-21.

[15] Sankaranarayanan R, Li Y, Greensmith DJ, Eisner DA, Venetucci L. Biphasic decay of the $\mathrm{Ca}$ transient results from increased sarcoplasmic reticulum Ca leak. J Physiol. 2015;3:611-23.

[16] Li L, Louch WE, Niederer SA, Aronsen JM, Christensen G, Sejersted OM, et al. Sodium accumulation in SERCA knockout-induced heart failure. Biophys J. 2012;102(9):2039-48.

[17] Bode EF, Briston SJ, Overend CL, O’Neill SC, Trafford AW, Eisner DA. Changes of SERCA activity have only modest effects on sarcoplasmic reticulum $\mathrm{Ca} 2+$ content in rat ventricular myocytes. J Physiol. 2011 Oct 1;589(Pt 19):4723-9.

[18] Wang J, Chan TO, Zhang X, Gao E, Song J, Koch WJ, et al. Induced overexpression of $\mathrm{Na}+\mathrm{Ca} 2+$ exchanger transgene: altered myocyte contractility, $[\mathrm{Ca} 2+]$ transients, SR Ca2+ contents, and action potential duration. 2009;297:590-601.

[19] Wright PT, Nikolaev VO, O’Hara T, Diakonov I, Bhargava A, Tokar S, et al. Caveolin-3 regulates compartmentation of cardiomyocyte beta2-adrenergic receptor-mediated cAMP signaling. J Mol Cell Cardiol. 2014;67:38-48.

[20] del Monte F, Harding SE, Schmidt U, Matsui T, Kang ZB, Dec GW, et al. Restoration of contractile function in isolated cardiomyocytes from failing human hearts by gene transfer of SERCA2a. Circulation. 1999;100(23):2308-11.

Address for correspondence.

María Teresa Mora

Centro de Investigación e Innovación en Bioingeniería (Ci2B)

Universitat Politècnica de València

Camino de Vera s/n Valencia 46022, Spain

mamofe@doctor.upv.es 